\title{
HIDROCARBONETOS POLICÍCLICOS AROMÁTICOS: CORRELAÇÃO ENTRE TEMPO DE USO DO ELETROCAUTÉRIO E TEMPO CIRÚRGICO*
}

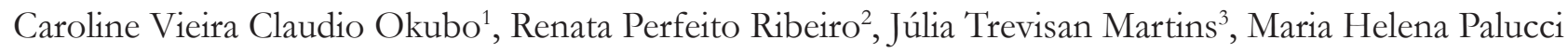 \\ Marziale $^{4}$
}

\begin{abstract}
RESUMO: Objetivou-se identificar concentrações de hidrocarbonetos policíclicos aromáticos provenientes da fumaça do eletrocautério nas salas operatórias, correlacionando estas concentrações com o tempo de uso do eletrocautério e cirúrgico. Trata-se de um estudo de campo, transversal e quantitativo, realizado em 50 atos cirúrgicos do aparelho digestivo, entre abril e julho de 2015, em hospital universitário no estado do Paraná. Foram coletados hidrocarbonetos por meio de uma bomba de sucção e determinados por cromatografia liquida. Estes compostos foram encontrados com média de $0,0058 \mathrm{mg} \cdot \mathrm{m}^{-3}$ e mediana de $0,0049 \mathrm{mg} \cdot \mathrm{m}^{-3}$. O tempo médio cirúrgico e de uso do eletrocautério foram de 136 minutos e 220,5 segundos, respectivamente. O teste de correlação de Spearman foi de -0,512 entre as variáveis concentrações e tempo cirúrgico e de -0,183 entre as concentrações e tempo de uso do eletrocautério. Conclui-se que existem hidrocarbonetos e há baixa correlação entre a produção destes compostos e tempo de uso do eletrocautério.
\end{abstract}

DESCRITORES: Exposição ocupacional; Poluentes ocupacionais do ar; Eletrocirurgia; Equipamentos de segurança.

\section{POLYCYCLIC AROMATIC HYDROCARBONS: CORRELATION BETWEEN THE TIME OF ELECTROCAUTERY USE AND SURGICAL TIME}

\begin{abstract}
The objective was to identify concentrations of polycyclic aromatic hydrocarbons produced by electrocautery smoke in operating rooms, correlating these concentrations with time of electrocautery and surgical use. This is a cross-sectional and quantitative field study carried out in 50 gastrointestinal surgical procedures, between April and July 2015, in a university hospital in the state of Paraná. Hydrocarbons were collected using a suction pump and measurements were determined through liquid chromatography. These compounds were found to have an average of $0.0058 \mathrm{mg} \cdot \mathrm{m}^{-3}$ and a mean of $0.0049 \mathrm{mg} \cdot \mathrm{m}^{-3}$. The mean surgical time and electrocautery use were 136 minutes and 220.5 seconds, respectively. The Spearman correlation test was -0.512 between concentration variables and surgical time, and -0.183 between the concentrations and electrocautery use. The conclusion was the presence of hydrocarbons and a low correlation between the production of these compounds and the electrocautery usage time. DESCRIPTORS: Occupational exposure; Occupational air pollutants; Electrosurgery; Protective devices.
\end{abstract}

\section{HIDROCARBUROS POLICÍCLICOS AROMÁTICOS: CORRELACIÓN ENTRE TIEMPO DE USO DEL ELECTROCAUTERIO Y TIEMPO QUIRÚRGICO}

\begin{abstract}
RESUMEN: Se objetivó identificar concentraciones de hidrocarburos policíclicos aromáticos derivados del humo del electrocauterio en quirófanos, correlacionando las concentraciones con el tiempo de uso del electrocauterio y quirúrgico. Estudio de campo, transversal, cuantitativo, realizado en 50 actos quirúrgicos del aparato digestivo, entre abril y julio de 2015, en hospital universitario del estado de Paraná. Fueron recolectados hidrocarburos mediante una bomba de succión, y determinados por cromatografía líquida. Los compuestos fueron encontrados con promedio de $0,0058 \mathrm{mg} . \mathrm{m} 3$ y mediana de $0,0049 \mathrm{mg} . \mathrm{m} 3$. El tiempo promedio quirúrgico y de uso del electrocauterio fue de 136 minutos y 220,5 segundos respectivamente. El test de correlación de Spearman fue de -0,512 entre variables concentraciones y tiempo quirúrgico, y de -0,183 entre las concentraciones y tiempo de uso del electrocauterio. Se concluye en que existen hidrocarburos y hay baja correlación entre la producción de tales compuestos y tiempo de uso del electrocauterio.
\end{abstract}

DESCRIPTORES: Exposición Profesional; Contaminantes Ocupacionales del Aire; Electrocirugía; Equipos de Seguridad.

\footnotetext{
*Artigo extraído da dissertação intitulada: "Hidrocarbonetos policíclicos aromáticos produzidos pela fumaça do eletrocautério: risco químico à equipe intraoperatória". Universidade Estadual de Londrina, 2016.
}

${ }^{1}$ Enfermeira. Mestre em Enfermagem. Hospital do Coração de Londrina. Londrina, PR, Brasil.

${ }^{2}$ Enfermeira. Pós-Doutoranda em Enfermagem. Docente de Enfermagem e do Programa de Pós-Graduação em Enfermagem da Universidade Estadual de Londrina. Londrina, PR, Brasil.

${ }^{3}$ Enfermeira. Doutora em Enfermagem. Docente de Enfermagem e do Programa de Pós-Graduação em Enfermagem da Universidade Estadual de Londrina. Londrina, PR, Brasil.

${ }^{4}$ Enfermeira. Pós-Doutora em Enfermagem. Docente de Enfermagem e do Programa de Pós-Graduação em Enfermagem da Universidade de São Paulo. Ribeirão Preto, SP, Brasil.

Autor Correspondente:

Caroline Vieira Claudio Okubo

Hospital do Coração de Londrina

R. Raposo Tavares, 442 - 86010-580 - Londrina, PR, Brasil

E-mail: caroline.vieirac@gmail.com
Recebido: 09/01/2017

Finalizado: $13 / 07 / 2017$ 


\section{INTRODUÇÃO}

O centro cirúrgico (CC) pode ser considerado como um setor peculiar em relação a outros setores da instituição hospitalar, por se tratar de um ambiente propenso a diversos riscos ocupacionais para os trabalhadores de saúde. Dentre os riscos presentes no CC, tem-se o risco químico caracterizado pela fumaça cirúrgica gerada pelo uso do eletrocautério.

O eletrocautério utiliza-se de corrente elétrica para o rompimento e hemostasia dos tecidos em diversas especialidades cirúrgicas. Dentre suas vantagens, tem-se a redução do tempo cirúrgico ${ }^{(1)}$.

Entretanto, entre as desvantagens, o uso do referido aparelho produz fumaça cirúrgica gerada pelo aquecimento do tecido e pela vaporização dos componentes celulares, ocasionando odor fétido para a equipe e diminuindo a visibilidade das cirurgias videolaparoscópicas ${ }^{(2-4)}$.

Essa fumaça cirúrgica é formada por vapor de água e compostos químicos ${ }^{(5-6)}$, incluindo os hidrocarbonetos policíclicos aromáticos (HPA) e os óxidos de carbono ${ }^{(5,7-8)}$. Também podem existir microorganismos na fumaça como o Mycobacterium tuberculosis e o vírus da imunodeficiência humana (HIV) ocasionado risco de infecção ainda não mensurado ${ }^{(6,9)}$. As referidas substâncias químicas podem ocasionar desde efeitos no sistema respiratório como ardor faríngeo e congestão nasal até câncer ao organismo humano ${ }^{(1,10)}$.

Dentre os compostos mencionados chama-se atenção para os HPA, os quais são compostos orgânicos formados por anéis aromáticos, carbono e hidrogênio ${ }^{(11)}$, com efeitos na pele, fígado e sistema imunológico, além de serem carcinogênicos ${ }^{(10)}$.

Agência americana afirmou que não há estudos disponíveis sobre os efeitos dos HPA no organismo humano baseados em níveis de concentrações específicos ${ }^{(12)}$, enfatizando a importância da realização de estudos que façam correlação entre as concentrações de HPA e os efeitos dessas concentrações no organismo humano.

O referido composto foi encontrado na fumaça do eletrocautério em estudos internacionais ${ }^{(5,13-15)}$, entretanto ainda são escassos estudos, principalmente nacionais, que abordam a presença dos compostos químicos na fumaça cirúrgica ${ }^{(16)}$, além daqueles que correlacionem o tempo de uso do eletrocautério e o tempo cirúrgico.

Assim, estudos afirmam que, quando se faz uso do eletrocautério, as medidas de proteção são essenciais para minimização dos riscos ocupacionais e proteção da equipe intraoperatória, exposta diariamente à fumaça cirúrgica. Precisam ser utilizados nos CC sistemas de exaustão local de fumaça próximos à emissão da mesma ${ }^{(17)}$, sistemas de ventilação eficientes ${ }^{(18)}$, além de equipamentos de proteção individual para a equipe intraoperatória, como a máscara respiratória $\mathrm{N}-95^{(17,19)}$ e óculos de proteção $^{(18)}$.

Entretanto, são escassas as legislações e recomendações internacionais de saúde ocupacional que regulamentem o uso de medidas de proteção nos atos cirúrgicos frente ao uso do eletrocautério no CC, como da National Institute for Occupational Safety and Health, Association of Perioperative Registered Nurses e da Nova Gales do Sul (Austrália) ${ }^{(17-19)}$, não sendo claras sobre o uso dessas medidas em relação ao tipo de cirurgia (aberta ou videolaparoscópica), especialidade cirúrgica, tempo cirúrgico e tempo de uso do eletrocautério. Além de que essas legislações/recomendações internacionais não legislam sobre o Brasil.

Ao analisarmos o descrito acima, frente às metas da Agenda 2030 da Organização das Nações Unidas para o Desenvolvimento Sustentável, uma delas é proteger os trabalhadores e promover ambientes de trabalho seguros e protegidos para todos os trabalhadores ${ }^{(20)}$. Esta meta e a escassez de estudos que façam a correlação entre os HPA, tempo cirúrgico e tempo de uso do eletrocautério, objetivou, neste estudo, responder aos seguintes questionamentos: Existem HPA no ar das salas operatórias? As concentrações dos HPA aumentam proporcionalmente ao uso do eletrocautério e ao tempo cirúrgico?

Diante do exposto, buscou-se identificar as concentrações de HPA provenientes da fumaça do eletrocautério nas salas operatórias e correlacionar as concentrações destes compostos com o tempo de uso do eletrocautério e o tempo cirúrgico. 
Pesquisa de campo, exploratória, transversal com abordagem quantitativa, desenvolvida em um CC de um hospital universitário de grande porte localizado no estado do Paraná, Brasil. O referido CC conta com sete salas operatórias nas quais são realizadas em média 700 cirurgias eletivas, de urgência e de emergência por mês em diversas especialidades, é formado por 262 trabalhadores de saúde e pósgraduandos lato sensu.

Para esta pesquisa foram escolhidas as cirurgias do aparelho digestivo, pois estas acontecem diariamente, em média duas cirurgias, fazendo uso do eletrocautério. Esta especialidade cirúrgica realiza em média 30 cirurgias por mês.

Os critérios de inclusão foram: cirurgias do aparelho digestivo eletivas ou de urgência com uso do eletrocautério durante o ato cirúrgico. Assim, a amostra intencional deste estudo contou com 50 cirurgias. O critério de exclusão foi cirurgia de emergência, devido à limitação em montar os aparelhos em tempo hábil, para o início da cirurgia e da coleta de dados.

O período de coleta ocorreu de abril a julho de 2015, nos turnos matutino, vespertino e noturno, sendo utilizado um formulário para a coleta de dados contendo as seguintes variáveis: caracterização da cirurgia e do uso do eletrocautério.

Uma bomba de sucção a vácuo da marca ASF Thomas ${ }^{\circledast}$ e do modelo $D$-82178 Puchhe im foi utilizada para coletar os HPA do ar das salas operatórias. Na referida bomba, por meio de um prolongamento de plástico, foram conectadas a cada ato cirúrgico seringas de cinco mililitros que continham uma resina da marca comercial Amberlite ${ }^{\circledast}$ XAD4 para a impregnação dos HPA, um filtro para a passagem dos HPA apenas na forma gasosa e uma espuma de polipropileno para fixação da resina XAD4 no cartucho.

A referida bomba esteve localizada na altura da área respiratória dos trabalhadores, especificamente a sete centímetros do campo operatório, permanecendo ligada sob a vazão de 120 litros por hora a cada ato cirúrgico. Os tempos cirúrgicos e uso do eletrocautério foram cronometrados por um cronômetro manual e digital.

Após a coleta, os cartuchos foram desconectados da bomba de sucção, guardados de forma apropriada e acondicionados, sob refrigeração, no laboratório de análise dos componentes do ar de uma universidade pública do Paraná. Posteriormente, foram realizadas as extrações das concentrações dos HPA da resina XAD4 e suas concentrações foram determinadas por Cromatografia Líquida de Alta Eficiência no referido laboratório.

Os resultados obtidos foram digitados na planilha Excel ${ }^{\circledast} 2010$ e analisados quantitativamente pelo software Statistical Package for the Social Sciences (SPSS) versão 20.0. A análise foi realizada de forma descritiva e analítica. Na descritiva, foram utilizadas medidas de tendência central e dispersão (média, desvio padrão, mediana, mínimo e máximo). Tratando-se da análise analítica, foi utilizado o teste de Shapiro-Wilk e o coeficiente de correlação de Spearman. Adotou-se nível de significância de $p<0,05$.

Em todas as fases desta pesquisa foram respeitados os aspectos éticos, sendo aprovado em setembro de 2014 pelo Comitê de Ética em Pesquisa envolvendo Seres Humanos da instituição do estudo, conforme parecer n. ${ }^{\circ} 785.868$.

\section{RESULTADOS}

A partir das 50 amostras analisadas, 17 (34\%) foram colecistectomias, sendo 27 (54\%) cirurgias abertas e $23(46 \%)$ videolaparoscópicas. Nestas cirurgias estavam presentes 62 trabalhadores de saúde, sendo 25 $(40,3 \%)$ do sexo feminino e 37 (59,7\%) do masculino. Destes, 11 (17,7\%) eram técnicos em enfermagem, seis $(9,7 \%)$ auxiliares em enfermagem, quatro $(6,5 \%)$ residentes em enfermagem perioperatória, seis $(9,7 \%)$ anestesiologistas, $11(17,7 \%)$ residentes em anestesiologia, nove (14,5\%) cirurgiões geral e 15 $(24,2 \%)$ residentes em cirurgia geral.

Os HPA foram encontrados em $100 \%$ das amostras, em todas as salas operatórias, cuja concentração 
média foi de $0,0058 \mathrm{mg} \cdot \mathrm{m}^{-3}$ com desvio padrão de $\pm 0,0049 \mathrm{mg} \cdot \mathrm{m}^{-3}$.

A Figura 1 mostra o histograma da frequência das concentrações dos HPA e suas concentrações.

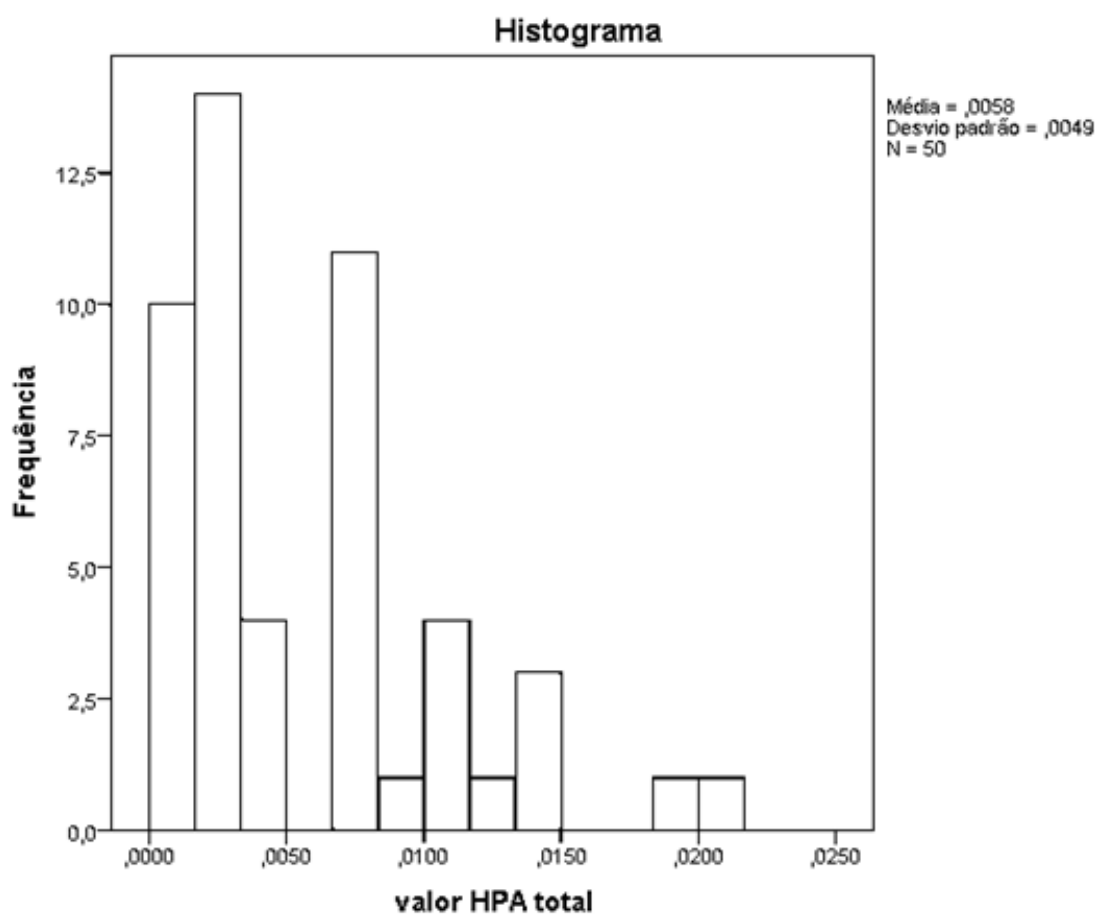

Figura 1 - Frequência das concentrações dos hidrocarbonetos policíclicos aromáticos. Londrina, PR, Brasil, 2017

A média do tempo total cirúrgico foi de 136 minutos com desvio padrão de \pm 84 minutos. Tratandose do uso do eletrocautério, a média foi de 220,5 segundos com desvio padrão de $\pm 226,4$ segundos.

Na Tabela 1, apresentam-se as estatísticas descritivas das concentrações dos HPA, tempo total cirúrgico e tempo de uso do eletrocautério.

Tabela 1 - Valores médios, mínimos, máximos, medianas e desvio padrão das concentrações dos HPA, tempo total cirúrgico e tempo de uso do eletrocautério detectados durante os atos cirúrgicos $(n=50)$. Londrina, $P R$, Brasil, 2017

\begin{tabular}{lcccc} 
Variáveis & Mediana & $\begin{array}{c}\text { Média } \pm \\
\text { desvio padrão }\end{array}$ & $\begin{array}{c}\text { Valores } \\
\text { mínimos }\end{array}$ & $\begin{array}{c}\text { Valores } \\
\text { máximos }\end{array}$ \\
\hline${\text { HPA* total }\left[\mathrm{mg} \cdot \mathrm{m}^{-3} \text { ] }\right.}$ & 0,005 & $0,0058 \pm$ & 0,000 & 0,021 \\
& & 13,0049 & 30 & 443 \\
\hline Tempo cirúrgico (minutos) & 113 & $136 \pm 84$ & 13 & 1160
\end{tabular}

* HPA - hidrocarbonetos policíclicos aromáticos

Constatou-se, por meio do teste de Spearman, que existe associação negativa entre os HPA e o tempo cirúrgico $(-0,512)$ e entre os HPA e o tempo de uso do eletrocautério $(-0,183)$. 


\section{DISCUSSÃO}

A presença de concentrações dos HPA, constatada neste estudo, no ar das salas operatórias tornase preocupante, visto que a equipe intraoperatória está exposta constantemente ao risco químico relacionado à fumaça do eletrocautério. Diversas categorias profissionais estão expostas à referida fumaça, sendo que nos Estados Unidos da América, aproximadamente 500.000 trabalhadores estão expostos por ano, incluindo cirurgiões, enfermeiros e instrumentadores cirúrgicos ${ }^{(21)}$.

A fumaça do eletrocautério e seu efeito na saúde humana têm sido o foco dos estudos. Atualmente, estudos têm sido publicados abordando não somente as consequências da queimadura da fumaça para o trabalhador e o risco de explosões nas salas operatórias ${ }^{(22)}$, mas também estudos que buscam mensurar os compostos da fumaça e os efeitos no organismo humano ${ }^{(5,7,13)}$. Entretanto, estes estudos ainda não são conclusivos, mas há forte evidência científica dos malefícios para o ser humano, incluindo a toxicidade na mucosa respiratória ${ }^{(1,23)}$.

A equipe intraoperatória é uma categoria que está exposta a pequena quantidade de fumaça, porém por um tempo prolongado(1). Assim, estes trabalhadores estão expostos constantemente aos HPA encontrados no presente estudo.

As concentrações dos HPA aumentam aproximadamente em 40 a 100 vezes no ar das salas operatórias após o uso do eletrocautério(7). Dessa forma, HPA prévios podem existir nas salas, mas seu aumento evidenciado no referido estudo confirma que os HPA são gerados pelo uso do eletrocautério.

Agência internacional de pesquisa sobre o câncer afirma que o HPA benzo(a)pireno é carcinógeno humano, entretanto a maioria dos hidrocarbonetos ainda são prováveis carcinógenos humanos, pois a evidência cancerígena em humanos ainda é limitada, sendo confirmada apenas em animais experimentais ${ }^{(11)}$. Estudo afirmou que existe risco de câncer para os cirurgiões frente aos HPA gerados pela fumaça cirúrgica, e esse risco correspondeu a $0,1 \%{ }^{(7)}$.

Assim como neste estudo, HPA foram encontrados em estudos transversais realizados em diversos países como na Alemanha, Suécia, Reino Unido e na Suíça, incluindo cirurgias abertas e videolaparoscópicas ${ }^{(5,13-15)}$.

Neste estudo maiores valores de HPA se concentraram no começo da cirurgia, conforme apresentada a figura 1. Os valores médios das concentrações de HPA, em 0,0058 com desvio padrão de 0,0049 mg.m 3 , foram maiores que a de outro estudo realizado na China que identificou valores médios de 0,0014 com desvio padrão de $0,0005 \mathrm{mg} \cdot \mathrm{m}^{-3}$ e média de 0,0010 e desvio padrão de $0,0006 \mathrm{mg} . \mathrm{m}^{-3}$, cuja coleta foi realizada próxima ao cirurgião e próxima ao anestesista ${ }^{(7)}$.

O tempo médio cirúrgico deste estudo, 136 minutos, assemelhou-se a outro estudo realizado com 15 laparotomias as quais apresentaram média de 143,3 minutos para o tempo cirúrgico ${ }^{(24)}$, entretanto a mediana, 113 minutos, diferenciou-se de outro estudo que foi de 614 minutos nas cirurgias de peritonectomia ${ }^{(14)}$.

Neste estudo a média do tempo de uso do eletrocautério, 220,5 segundos, foi menor que a de outros estudos realizados em mastectomias que identificaram 1.986 e 1.650 segundos $^{(7,25)}$. A mediana, 138,5 segundos, também foi menor, pois estudo internacional identificou 4.110 segundos ${ }^{(8)}$.

Um estudo mostrou que há correlação entre a quantidade de HPA produzidos e o sangramento do tecido ${ }^{(14)}$. Neste estudo, o teste de Spearman mostrou que há correlação média e negativa entre as concentrações dos HPA detectadas e o tempo de cirurgia $(-0,512)$. Também há correlação baixa e negativa entre estas concentrações e o tempo de uso do eletrocautério $(-0,183)$. Assim, estas variáveis são inversamente proporcionais, pois com o decorrer da cirurgia e do uso do eletrocautério, há diminuição dos HPA presentes no local da coleta, que foi a sete centímetros da abertura do campo operatório.

Supõe-se assim que, à medida que a cirurgia se desenvolve, os HPA de dissipam pela sala, sendo um risco não apenas para a equipe cirúrgica, próxima ao campo operatório, mas também para o circulante de sala ou outro trabalhador que está afastado do campo operatório. 
Frente a esta constatação, é necessário o uso de equipamentos de proteção individual por todos os trabalhadores da equipe intraoperatória. Entre eles está o uso da máscara N95, N99 ou N100 as quais oferecem eficácia entre $95 \%$ e 99,9\% $\%^{(17-18,26-27)}$.

As máscaras cirúrgicas, utilizadas atualmente nas salas operatórias ${ }^{(28)}$, não são adequadas, visto que não protegem corretamente os trabalhadores frente à presença de poluentes químicos gerados pela fumaça ${ }^{(29)}$. Aliado ao uso das máscaras respiratórias, faz-se necessário o uso dos óculos de proteção para proteção da mucosa ocular(18).

A fumaça cirúrgica precisa ser removida por sistemas de evacuação portátil que a recolhem e a encaminham para ser filtrada, tanto nos procedimentos abertos como nos videolaparoscópicos. Entre eles estão a caneta eletrocirúrgica monopolar com tubo de evacuação acoplado e o evacuador de fumaça portátil ${ }^{(30)}$.

Apesar da importância das medidas preventivas mencionadas, autor afirma que há atitude de desprezo frente aos riscos da inalação da fumaça cirúrgica ${ }^{(30)}$. Esta prática pode estar relacionada à falta de conhecimento pelo trabalhador ou à falta de políticas educativas que incentivem a prevenção.

Diante do exposto, é primordial que os trabalhadores da equipe intraoperatória utilizem as medidas preventivas mencionadas em todos os atos cirúrgicos, pois há correlação baixa entre o tempo cirúrgico e o tempo de uso do eletrocautério com relação aos HPA, constituindo em risco químico para os referidos trabalhadores.

Como limitações deste estudo, menciona-se o tipo do estudo, exploratório, não sendo possível sua aplicação a outros campos de estudo. Outra limitação foi a coleta dos HPA ter sido realizada apenas próximo ao campo operatório, insuficiente para mensurar o risco para o circulante de sala, que fica mais afastado do campo cirúrgico.

Os resultados deste estudo colaboram para avanços na área de Saúde Pública, Saúde do Trabalhador e para o Centro Cirúrgico, visto que alerta a comunidade científica frente aos riscos provenientes da fumaça do eletrocautério e possibilita que programas de prevenção e legislações sejam implementados a fim de evitar o adoecimento do trabalhador.

\section{CONCLUSÃO}

Os resultados desta pesquisa comprovam que existem HPA presentes no ar das salas operatórias, proveniente do uso da fumaça do eletrocautério, tanto nas cirurgias abertas como nas videolaparoscópicas, sendo que as maiores concentrações se concentraram no início das cirurgias.

Há correlação média e baixa inversamente proporcional entre os HPA e os tempos cirúrgicos e os tempos de uso do eletrocautério, ou seja, os HPA não aumentam proporcionalmente frente ao uso do eletrocautério e ao tempo cirúrgico. Assim, há evidência que os HPA se dissipam pelo ar das salas operatórias.

O uso de equipamentos de proteção individual como as máscaras respiratórias, óculos de proteção e os evacuadores portáteis são fortes aliados no combate à inalação da fumaça cirúrgica e propiciadores de ambiente ocupacional seguro para todos os trabalhadores da equipe intraoperatória.

\section{REFERÊNCIAS}

1. Navarro-Meza MC, González-Baltazar R, Aldrete-Rodríguez MG, Carmona-Navarro DE, López-Cardona MG. Síntomas respiratorios causados por el uso del electrocauterio en médicos en formación quirúrgica de un hospital de México. Rev Peru Med Exp Salud Publica. [Internet] 2013;30(1) [acesso em 05 ago 2016]. Disponível: http://dx.doi.org/10.1590/S1726-46342013000100008.

2. Al Sahaf OS, Vega-Carrascal I, Cunningham FO, McGrath JP, Bloomfield FJ. Chemical composition of smoke produced by high-frequency electrosurgery. Ir J Med Sci. [Internet] 2007;176(229) [acesso em 05 ago 2016]. Disponível: http://dx.doi.org/10.1007/s11845-007-0068-0. 
3. Ball K. Surgical smoke evacuation guidelines: compliance among perioperative nurses. AORN J. [Internet] 2010;92(2) [acesso em 10 ago 2016]. Disponível: http://dx.doi.org/10.1016/j.aorn.2009.10.026.

4. Weld KJ, Dryer S, Ames CD, Cho K, Hogan C, Lee M, et al. Analysis of Surgical Smoke Produced by Various Energy-Based Instruments and Effect on Laparoscopic Visibility. J Endourol. [Internet] 2007;21(3) [acesso em 12 ago 2016]. Disponível: http://dx.doi.org/10.1089/end.2006.9994.

5. Gianella M, Hahnloser D, Rey JM, Sigrist MW. Quantitative chemical analysis of surgical smoke generated during laparoscopic surgery with a vessel-sealing device. Surg Innov. [Internet] 2014;21(2) [acesso em 13 ago 2016]. Disponível: http://dx.doi.org/10.1177/1553350613492025.

6. Eickman IU, Falcy M, Fokuhl I, Rüegger M. International Section of the ISSA on prevention of occupational risks in health services. Surgical smoke: Risks and preventive measures. Germany: International Social Security Association (ISSA); 2012 [acesso em 10 set 2016]. Disponível: http://prevencion.umh.es/files/2012/04/2-surgical_ smoke.pdf.

7. Tseng HS, Liu SP, Uang SN, Yang LR, Lee SC, Liu YJ, et al. Cancer risk of incremental exposure to polycyclic aromatic hydrocarbons in electrocautery smoke for mastectomy personnel. World J Surg Oncol. [Internet] 2014;12(31) [acesso em 02 jul 2016]. Disponível: http://dx.doi.org/10.1186/1477-7819-12-31.

8. Choi SH, Kwon TG, Chung SK, Kim TH. Surgical smoke may be a biohazard to surgeons performing laparoscopic surgery. Surg Endosc. [Internet] 2014;28(8) [acesso em 04 jul 2016]. Disponível: http://dx.doi.org/10.1007/s00464014-3472-3.

9. Chowdhury KK, Meftahuzzaman SM, Rickta D, Chowdhury TK, Chowdhury BB, Ireen ST. Electrosurgical smoke: a real concern. Mymensingh Med J. [Internet] 2011;20(3) [acesso em 04 jul 2016]. Disponível: http://www. banglajol.info/index.php/MMJ/article/view/8339.

10. United States of America. United States Department of Health and Human Services. Agency for Toxic Substances and Disease Registry. Polycyclic Aromatic Hydrocarbons (PAHs). [Internet] 2011 [acesso em 25 mar 2016]. Disponível: http://www.atsdr.cdc.gov/substances/toxsubstance.asp?toxid=25.

11. World Health Organization (WHO). International Agency for Research on Cancer. IARC Monographs on the Evaluation of Carcinogenic Risks to Humans. Some Non-heterocyclic Polycyclic Aromatic Hydrocarbons and Some Related Exposures. [Internet] Lyon: IARC; 2010 [acesso em 24 maio 2016]. Disponível: https://monographs. iarc.fr/ENG/Monographs/vol92/mono92.pdf.

12. United States of America. United States Environmental Protection Agency. Polycyclic Aromatic Hydrocarbons (PAHs). [Internet] 2008 [acesso em 10 ago 2016]. Disponível: http://www3.epa.gov/epawaste/hazard/wastemin/ minimize/factshts/pahs.pdf.

13. Kisch T, Liodaki E, Kraemer R, Mailaender P, Brandenburger M, Hellwig V, et al. Electrocautery devices with feedback mode and teflon-coated blades create less surgical smoke for a quality improvement in the operating theater. Medicine. [Internet] 2015;94(27) [acesso em 05 jul 2016]. Disponível: http://doi.org/10.1097/ MD.0000000000001104.

14. Andréasson SN, Mahteme H, Sahlberg B, Anundi H. Polycyclic aromatic hydrocarbons in electrocautery smoke during peritonectomy procedures. J Environ Public Health. [Internet] 2012;2012(929053) [acesso em 05 jul 2016]. Disponível: http://dx.doi.org/10.1155/2012/929053.

15. Fitzgerald JE, Malik M, Ahmed I. A single-blind controlled study of electrocautery and ultrasonic scalpel smoke plumes in laparoscopic surgery. Surg Endosc. [Internet] 2012;26(2) [acesso em 06 jul 2016]. Disponível: http://dx.doi.org/10.1007/s00464-011-1872-1.

16. Tramontini CC, Galvão CM, Claudio CV, Ribeiro RP, Martins JT. Composição da fumaça produzida pelo bisturi elétrico: revisão integrativa da literatura. Rev. esc. enferm. USP. [Internet] 2016;50(1) [acesso em 04 mar 2016]. Disponível: http://dx.doi.org/10.1590/S0080-623420160000100019.

17. Association of Perioperative Registered Nurses. Recommended practices for electrosurgery. In: Perioperative Standards and Recommended Practices. Denver: AORN; 2012. p. 99-118.

18. New South Wales. Occupational Health \& Safety. Work Health and Safety - Controlling Exposure to Surgical Plume. [Internet] Sydney: Occupational Health \& Safety; 2015 [acesso em 15 ago 2016]. Disponível: http://www0. 
health.nsw.gov.au/policies/gl/2015/pdf/GL2015_002.pdf.

19. Spruce L, Braswell ML. Implementing AORN Recommended Practices for Electrosurgery. AORN J. [Internet] 2012;95(3) [acesso em 10 ago 2016]. Disponível: http://dx.doi.org/10.1016/j.aorn.2011.12.018.

20. United Nations. Objective 8. To promote sustained, inclusive and sustainable economic growth, full and productive employment and decent work for all. [Internet] 2014 [acesso em 10 ago 2016]. Disponível: https:// sustainabledevelopment.un.org/sdg8.

21. United States of America. United States Departmente of Labor. Occupacional Safety \& Health Administration. Safety and Health Topics. Laser/Electrosurgery Plume. [Internet] Washington: Occupational Safety \& Health Administration; 2016 [acesso em 15 ago 2016]. Disponível: https://www.osha.gov/SLTC/laserelectrosurgeryplume/.

22. Afonso CT, da Silva AL, Fabrini DS, Afonso CT, Côrtes MGW, Sant'Anna LL. Risco do uso do eletrocautério em pacientes portadores de adornos metálicos. ABCD, arq. bras. cir. dig. [Internet] 2010;23(3) [acesso em 12 set 2016]. Disponível: http://dx.doi.org/10.1590/S0102-67202010000300010.

23. Atar Y, Salturk Z, Kumral TL, Uyar Y, Cakir C, Sunnetci G, et al. Effects of Smoke Generated by Electrocautery on the Larynx. J Voice. [Internet] 2017;31(3) [acesso em 10 set 2016]. Disponível: http://dx.doi.org/10.1016/j. jvoice.2016.05.012.

24. Wu YC, Tang CS, Huang HY, Liu CH, Chen YL, Chen DR, et al. Chemical production in electrocautery smoke by a novel predictive model. Eur Surg Res. [Internet] 2011;46(2) [acesso em 09 set 2016]. Disponível: http://dx.doi. org/10.1159/000322855.

25. Lin YW, Fan SZ, Chang KH, Huang CS, Tang CS. A novel inspection protocol to detect volatile compounds in breast surgery electrocautery smoke. J Formos Med Assoc. [Internet] 2010;109(7) [acesso em 08 set 2016]. Disponível: http://dx.doi.org/10.1016/S0929-6646(10)60085-X.

26. Gao S, Koehler RH, Yermakov M, Grinshpun SA. Performance of Facepiece Respirators and Surgical Masks Against Surgical Smoke: Simulated Workplace Protection Factor Study. Ann Occup Hyg. [Internet] 2016;60(5) [acesso em 10 fev 2017]. Disponível: https://doi.org/10.1093/annhyg/mew006.

27. United States of America. Centers for Disease Control and Prevention. National Institute for Occupational Safety and Health (NIOSH). NIOSH Science Blog. N95 respirators and surgical masks. [Internet] 2015 [acesso em 19 ago 2016]. Disponível: http://blogs.cdc.gov/niosh-science-blog/2009/10/n95.

28. Coffey CC, Lawrence RB, Campbell DL, Zhuang Z, Calvert CA, Jensen PA. Fitting characteristics of eighteen N95 filtering-facepiece respirators. J Occup Environ Hyg. [Internet] 2004;1(4) [acesso em 04 set 2016]. Disponível: http://dx.doi.org/10.1080/15459620490433799.

29. Agência Nacional de Vigilância Sanitária (ANVISA). Cartilha de Proteção Respiratória contra Agentes Biológicos para Trabalhadores de Saúde. Brasília: ANVISA; 2009.

30. Schultz L. An Analysis of Surgical Smoke Plume Components, Capture, and Evacuation. AORN J. [Internet] 2014;99(2) [acesso em 10 ago 2016]. Disponível: http://dx.doi.org/10.1016/j.aorn.2013.07.020. 